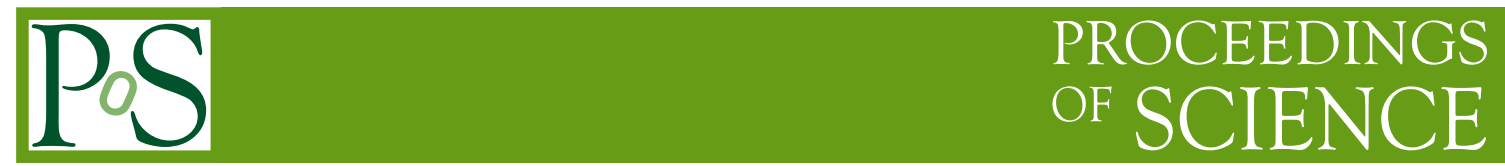

\title{
Higgs production at the Tevatron and LHC
}

\author{
Giampiero Passarino* ${ }^{\dagger}$ \\ Dipartimento di Fisica Teorica, Università di Torino, Italy \\ INFN, Sezione di Torino, Italy \\ E-mail: giampiero@to.infn.it
}

Status of the art in the calculation of Higgs cross sections and their theoretical and parametric errors is summarized.

35th International Conference of High Energy Physics - ICHEP2010,

July 22-28, 2010

Paris France

\footnotetext{
* Speaker.

†Work supported by the European Community's Marie-Curie Research Training Network under contract MRTNCT-2006-035505 'Tools and Precision Calculations for Physics Discoveries at Colliders'
} 


\section{Introduction}

In this talk we report on work done within the Higgs XS Working Group ${ }^{1}$ and summarize Higgs boson production and decay from Tevatron to LHC [1, 2, 3].

Common and correlated theoretical inputs (cross sections, PDFs, SM inputs, etc.) require the highest standards on the theoretical side. The goal of the WG has been to give precise common inputs to the experiments to facilitate the combination. The major effort has been devoted to discuss computation of cross sections and branching ratios for the SM Higgs and for the Minimal Supersymmetric Standard Model (MSSM) Higgs bosons, including the still-remaining theoretical uncertainties.

The Higgs XS WG is now moving into second phase, beyond fully inclusive quantities. Here we briefly summarize the roadmap. Definition of control - region is one place where the theoretical input will be mostly relevant: for any given observable the strategy will be to invert cuts and go from signal enhancement to background enhancement. Then one uses data to normalize background and theory to compute changes in background when inverting cuts. Thus a common recipe is needed for extrapolation from control to signal regions (e.g. QCD scales, PDF error, etc.) When talking about systematic uncertainties we have the theory driven ones: if total cross sections yield normalization, differential ones are relevant for the shape of discriminating quantities.

New activities require an unprecedented precision and the area of Monte Carlo (MC) at nextto-leading order (NLO) will become important as next step for exclusive calculations requiring differential distributions for Higgs signal, for example Higgs $p_{T}$, comparison between lowest-order (LO) Parton Shower MC and NLO MC, normalization to next-to-next leading order (NNLO). One last set of problems to solve: the extension of theoretical uncertainty (THU) definition in exclusive Higgs cross sections and the inclusion of signal and background interference effects. The latter, at the moment, is mostly done with LO MC. Then, how to extrapolate to higher order (e.g. $q q / g g \rightarrow$ $\gamma \gamma, q q / g g \rightarrow W W / Z Z \rightarrow l v l v$ etc.)?

Clearly, the reliability of results needs theoretical input and we have proposed to study theoretical errors of SM backgrounds to Higgs search with common ATLAS and CMS cuts. In the following sections we discuss few (selected) items, status of gluon - gluon fusion, theoretical uncertainties and pseudo-observables at hadron colliders that represent a tiny fraction of the huge activity in the Higgs XS WG.

\section{The importance of being $\mathrm{N}^{n} \mathrm{LO}$}

Recent years have seen an impressive amount of new results at $\mathrm{N}^{n} \mathrm{LO}$, an important step because NLO is the first order where reliable predictions can be obtained but NNLO is the first order at which a reliable estimate of the error can be given, see Ref. [4, 5, 6, 7]. Usually these calculations are (fully) inclusive, but experiments have finite acceptances and a considerable amount of progress has been achieved also for exclusive quantities, e.g. differential K-factors.

\footnotetext{
${ }^{1}$ https://twiki.cern.ch/twiki/bin/view/LHCPhysics
} 


\subsection{Theoretical uncertainties}

Here we present a short list of sources for theoretical uncertainties. For signal cross sections one has to include: parametric errors and their propagation; electroweak (EW) corr, e.g. renormalization scheme [8, 9]; QCD $\otimes / \oplus$ EW corr (factorized or added?) [10]; QCD scales (renormalization $\mu_{R}$, factorization $\mu_{F}$ ); here the main problem is the definition of a central value, the range of variation and the scan strategy; PDF uncertainties [11, 12]. For background treatment: LO $\times$ K-factor or NLO? Is there a practical way of including the interference of background with signal [13], etc.? It is worth nothing that THUs are $100 \%$ correlated between the two experiments.

The concept of THU and its use require few basic rules and an agreement within the community: sets of options in different calculations should be homogeneous; if one calculation includes a new option its physical origin should be motivated and its inclusion accepeted, in which case all codes should include it. If different calculations include homogeneous sets of options the difference beween central values should be considered with particular care, unless the central value itself reflects a specific choice for the preferred setup with different choices in different calculations. If all options, including the preferred setup, are congruent then differences in the central values cannot by justified by THU. We can ask the following question: is there a $\mu_{R}$ problem in QED? The answer is yes but, is it a problem? This time the answer is no because there is a physical subtraction point available, i.e. $q^{2}=0$. Next question would be: is there a $\mu_{R}$ in the EW theory? Yes, of course, but, once again physical input data are available. Do we have large logarithmic corrections surviving renormalization? Yes, we have, but there is a very simple solution: use $G_{F}$ - scheme and not $\alpha(0)$, i.e. resum the large logarithms.

What to do in QCD? Resummation is the keyword but, admittedly, it is not always available. Therefore, the most useful keyword will be minimization. To understand the problem consider a physical observable which is affected by (large) QCD corrections. Since we have no analogue of $G_{F}$ our LO calculation will always contain logarithms $\ln \left(s / \mu_{R}\right)$ where $s$ is the scale where we want to study the process. Ideally, one should find a scale $s_{0}$ where some data is available and renormalization means the replacement $\ln \left(s / \mu_{R}\right) \rightarrow \ln \left(s / s_{0}\right)$ and $s_{0}$ should not be far away from $s$. This is not (yet) possible in QCD, so the question will translate into, how do I choose $\mu_{R}$ ? The guideline will be set $\mu_{R}$ to $s$, or, in other words, make sure that you don't change much by going to the next order. This is easy in a one-scale process but in any multi-scale process one will have other additional large logarithms, say of argument $s / s^{\prime}$. What to do? Select $\mu_{R}$ and $\mu_{F}$, process by process, in such a way that when going from $\mathrm{N}^{n} \mathrm{LO}$ to $N^{n+1} \mathrm{LO}$ you minimize the effect of the new corrections. The recipe is the best simulation of a subtraction at some physical point close to the relevant scale. In jargon this is called dynamical scale.

Next, we show a nice example of THU, reflecting its statistical meaning. Consider gluongluon fusion, the optimal choice for a fixed-order calculation is to set the scale at $M_{H} / 2$, which is fully justified once we have NNL re-summation [14], Therefore, the practical recipe for a multi scale problem will be to select a dynamical scale for $\mu$ and to vary $\mu_{\min } \leq \mu \leq \mu_{\max }$, where the scale and its range of variation are selected to (reasonably) minimize large logarithmic corrections. An example, relevant for $\mathrm{LHC} @ 7 \mathrm{TeV}$, is given by the production cross section in gluon - gluon fusion at $M_{H}=165 \mathrm{GeV}$ showing perfect consistency, see Ref. [4, 15] and Ref. [6]

- De Florian, Grazzini $\sigma=8.45_{-0.66}^{+0.64}(\text { scale })_{-0.27}^{+0.33}\left(\mathrm{PDF}+\alpha_{s}\right)$ 
- Anastasiou et al. $\sigma=8.54_{-0.78}^{+0.64}(\text { scale })_{-0.28}^{+0.34}\left(\mathrm{PDF}+\alpha_{s}\right)$

\section{A new language?}

Higgs boson decays are considered, in the experimental analyses, as on-shell Higgs bosons decaying according to their BR's, including higher order effects. However, the quantities that can be directly measured in the (LHC) experiments are cross sections, asymmetries, etc., called "Realistic observables" (RO). In order to determine quantities like Higgs boson masses, partial widths or couplings from the RO a deconvolution procedure (unfolding some of the higher-order corrections, interference contributions etc.) has to be applied. These secondary quantities are called "pseudo observables" (PO) [16]. Therefore, the main question that we are going to address is about the meaning of any future comparison (theory versus data) where, for instance, $\Gamma(H \rightarrow \gamma \gamma)$ computed at $n$-loops is compared with something extracted from the data with much less precision and, sometimes, in a way that is not completely documented. Without loss of generality it will be useful to introduce an elementary glossary of terms: In conclusion, the only purpose of this Section has been

\begin{tabular}{|l|l|}
$\mathbf{R D}=$ & real data \\
$\mathbf{R O}=$ & $\begin{array}{l}\text { going from real data to distributions with cuts defines } \mathbf{R O}_{\text {exp }}, \\
\text { e.g. from diphoton pairs }(E, p) \text { to } \mathbf{M}(\gamma \gamma) \text {; given a model, e.g. SM, } \mathbf{R O}_{\text {th }} \text { can be computed } \\
\text { transform the universal intuition of a } Q F T \text {-non-existing quantity into an archetype, } \\
\text { e.g. } \sigma(g g \rightarrow H), \Gamma(H \rightarrow \gamma \gamma), \mathbf{R} \mathbf{O}_{\text {th }}\left(M_{H}, \Gamma(H \rightarrow \gamma \gamma), \ldots\right) \\
\text { fitted to } \mathbf{R O}_{\exp }\left(\text { e.g. } \mathbf{R O}_{\exp }=M(\gamma \gamma)\right) \text { defines and extracts } \mathbf{M}_{H} \text { etc. }\end{array}$ \\
\hline
\end{tabular}

to state the problem and the possible way to solutions, conventional but unique:

\begin{tabular}{|c|c|c|}
\hline on-shell $\rightarrow$ & $|\mathbf{H}>\rightarrow| \mathbf{f}>$ & $\leftarrow \quad$ does not exist \\
& $\downarrow$ & \\
well defined & $\leftarrow$ & $\mathrm{RO}_{\mathrm{f}}^{\text {th }}\left(\mu_{H} \Gamma\left(H_{c} \rightarrow f\right), \ldots\right)$ \\
$\mathrm{RO}^{\exp }$ & $\rightarrow$ & $\mu_{H}, \Gamma\left(H_{c} \rightarrow f\right) \leftarrow$ extracted \\
& & \\
\hline
\end{tabular}

\section{Conclusions}

In conclusion $\mathrm{N}^{n} \mathrm{LO}$ corrections are important, they are known is several cases, e.g. QCD up to NNLO for gluon-gluon fusion; refinements are available, e.g. resummation and EW effects, supporting the statement that theoretical predictions are well under control. Fully exclusive NLO (or higher) programs exist that allow us to compute corrections in the presence of cuts and the experimental collaborations are starting to use them.

Finally, we need a consistent definition of mass, width, couplings of the Higgs boson to publish results in such a way that theorists can later enter their general model parameters and see how well data constrain this model. 


\section{Acknowledgements}

I take this opportunity to acknowledge the precious guidance of A. Denner, S. Dittmaier, S. Farrington M. Felcini, M. Grazzini, C. Mariotti, F. Petriello, M. Spira, R. Tanaka.

\section{References}

[1] CDF and D0 Collaboration, Combined CDF and D0 Upper Limits on Standard Model Higgs- Boson Production with up to $6.7 \mathrm{fb}^{-1}$ of Data, arXiv: 1007.4587 [hep-ex] .

[2] C. ATLAS, ATLAS Sensitivity Prospects for Higgs Boson Production at the LHC Running at $7 \mathrm{TeV}$, Tech. Rep. ATL-PHYS-PUB-2010-009, CERN, Geneva, Jul, 2010.

[3] S. Abdullin et al., Summary of the CMS potential for the Higgs boson discovery, Eur. Phys. J. C39S2 (2005) 41-61.

[4] D. de Florian and M. Grazzini, Higgs production through gluon fusion: updated cross sections at the Tevatron and the LHC, Phys. Lett. B674 (2009) 291-294, arXiv: 0901.2427 [hep-ph] .

[5] C. Anastasiou, K. Melnikov, and F. Petriello, Fully differential Higgs boson production and the di-photon signal through next-to-next-to-leading order, Nucl. Phys. B724 (2005) 197-246, hep-ph/0501130.

[6] C. Anastasiou, G. Dissertori, and F. Stockli, NNLO QCD predictions for the $H$-> WW -> l l nu nu signal at the LHC, JHEP 09 (2007) 018, arXiv : 0707.2373 [hep-ph] .

[7] C. Anastasiou, G. Dissertori, F. Stoeckli, and B. R. Webber, $Q C D$ radiation effects on the $H->W W->l$ nu l nu signal at the LHC, arXiv:0801.2682 [hep-ph] .

[8] S. Actis, G. Passarino, C. Sturm, and S. Uccirati, NLO Electroweak Corrections to Higgs Boson Production at Hadron Colliders, Phys. Lett. B670 (2008) 12-17, arXiv: 0809.1301 [hep-ph ] .

[9] A. Denner and S. Dittmaier, The complex-mass scheme for perturbative calculations with unstable particles, Nucl. Phys. Proc. Suppl. 160 (2006) 22-26, arXiv: hep-ph / 0605312.

[10] C. Anastasiou, R. Boughezal, and F. Petriello, Mixed QCD-electroweak corrections to Higgs boson production in gluon fusion, JHEP 04 (2009) 003, arXiv: 0811.3458 [hep-ph] .

[11] PDF4LHC Steering Committee: http://hep.ucl.ac.uk/pdf 4 lhc.

[12] PDF4LHC Wiki:

https://wiki.terascale.de/index.php?title=PDF4LHC_WIKI.

[13] L. J. Dixon and M. S. Siu, Resonance-continuum interference in the di-photon Higgs signal at the LHC, Phys. Rev. Lett. 90 (2003) 252001, arXiv : hep-ph/ 0302233.

[14] S. Catani, D. de Florian, M. Grazzini, and P. Nason, Soft-gluon resummation for Higgs boson production at hadron colliders, JHEP 07 (2003) 028, hep-ph / 0306211.

[15] M. Grazzini, NNLO predictions for the Higgs boson signal in the $H->W W->\ln u \ln u$ and $H->Z Z->4 l$ decay channels, arXiv:0801.3232 [hep-ph] .

[16] G. Passarino, C. Sturm, and S. Uccirati, Higgs Pseudo-Observables, Second Riemann Sheet and All That, Nucl. Phys. B834 (2010) 77-115, arXiv:1001.3360 [hep-ph] . 